\title{
Autoradiographic study of the distribution of 55-iron(III)-EDTA chelate at the presence of humic substances in Triticum aestivum $L$.
}

\author{
Zimbovskaya M.M., Kulikova N.A., Filippova O.I., Pankratov D.A. \\ Lomonosov Moscow State University, Moscow, Russia, pankratov@radio.chem.msu.ru
}

Keywords: autoradiography, iron(III)-EDTA chelate, wheat

doi: 10.36291/HIT.2019.zimbovskaya.066

EDTA is one of the widely used ligands for delivering minerals to plants. It is known that EDTA-ion forms stable, highly soluble compounds with many metals in a wide $\mathrm{pH}$ range. This provides easy transport of mineral nutrients to plant tissue. Therefore, EDTA complexes, for example, with iron are often used in laboratory practice as a control when studying the absorption of other iron-containing preparations by plants or in agrochemistry production for the operational prevention of plant diseases. In the first case, sometimes, iron compounds in the presence of humic substances (HS) are considered as alternative iron-containing preparations [1]. In the second case, the contact of the iron(III)-EDTA chelate with the HS of the soil or substrate on which the cultivated plant handled is inevitable. In any case, participation and possible role of HS in the distribution of ironcontaining preparations general and, in particular, iron(III)-EDTA chelate are of interest.

Therefore, the aim of this work was to study the possible participation of HS coal in the distribution of iron(III)-EDTA chelate in the parts of wheat Triticum aestivum L. at root and foliar uptake. The autoradiographic method is studying the distribution of nuclides in a plant directly. It is based on the use of the sensitivity of some materials to radionuclide radiation. In the present work are used, BaFBr:Eu ${ }^{2+}$ luminescent screens in the Cyclone Storage System (Perkin Elmer) digital radiography system were used as a detector. As the detected radionuclide, 55-iron was used, which is characterized by optimal characteristics both in terms of radiation energy (mainly $\beta$-radiation - $231.4 \mathrm{keV}$ ) and half-life (2.73 y). With this radionuclide label iron(III)-EDTA chelate was synthesized, and the purity of which was confirmed by thin-layer chromatography. This radiation-emitting material was used to prepare four solutions: two for the experiment with root absorption, and two for the experiment with leaf absorption, differing between the pairs by the concentration of iron(III)-EDTA chelate (0.558 and $0.055 \mathrm{mg} / \mathrm{l}$, accordingly). In each pair, one solution contained only iron(III)-EDTA chelate, and in another potassium humate was additionally added to a concentration of $1000 \mathrm{mg} / \mathrm{l}$. The root uptake experiment was use 18 days of age wheat seedlings, which were placed for one day in vessels with a labeled solution. The leaf absorption experiment was carried out with seedlings of wheat, which were sprayed with a labeled solution and kept for 7 days. After germination, the plants were washed, dried and subjected to autoradiography. In total, 18 plants at root uptake and 16 plants at foliar uptake were studied. From the data obtained it follows that the presence of HS affects the distribution of iron(III)-EDTA chelate. Sic, the formation of "films" was observed the roots at accumulating the iron-containing preparation. HS contribute is evenly distributed iron contained preparation in the shoots at root uptake. In the absence of HS, the 55-iron is predominantly located at the points of the most intensive plant growth - at the tips of the roots, at the tips of the shoots, and also in the thickened lower part of the shoot. During foliar uptake of iron concentration is observed in the at root part of shoots, and in the presence of HS, the iron content is much higher. Thus, in the work the role of HS in the distribution of iron in such a strong complex compound as iron(III)-EDTA chelate is demonstrated.

\section{References}

1. Kulikova N.A. et al. // J. Agric. Food Chem. 2017. V. 65, № 51. P. 11157-11169. DOI: 10.1021/acs.jafc.7b03955. 